\title{
Revisión de los últimos eventos magmáticos del Cenozoico del sector norte-central de la Sierra Madre del Sur y su posible conexión con el subsuelo profundo de la Cuenca de México
}

\author{
Enrique Alejandro González Torres ${ }^{1, *}$, Dante J. Morán Zenteno ${ }^{2}$, Laura Mori ${ }^{1}$, \\ Barbara M. Martiny ${ }^{2}$ \\ ${ }^{1}$ División de Ingeniería en Ciencias de la Tierra, Facultad de Ingeniería, Universidad Nacional Autónoma de México, Ciudad \\ Universitaria, Código Postal 04510, México, D.F., México \\ ${ }^{2}$ Departamento de Geoquímica, Instituto de Geología, Universidad Nacional Autónoma de México, Ciudad Universitaria, Código \\ Postal 04510, México, D.F., México. \\ *eagtgaia@unam.mx
}

\begin{abstract}
Resumen
En el presente trabajo se revisa la posible correlación de las unidades del subsuelo profundo de la Cuenca de México (CM) que forman las sucesiones inferiores de pozos de perforación, con el registro estratigráfico del Cenozoico anterior a la Faja Volcánica Trans-Mexicana (FVTM) expuesto en la parte norte de la Sierra Madre del Sur (SMS).

En años recientes se han realizado estudios estratigráficos y geocronológicos de algunos centros eruptivos del sector norte-central de la SMS, que forman los campos volcánicos de Taxco y Huautla, y la caldera de Tilzapotla. En dichos campos se encuentra el registro de los últimos episodios volcánicos anteriores a la formación de la FVTM, y que constituye el extinto arco del Paleógeno de la SMS. Con base en las analogías litológicas, la posición estratigráfica y el espesor del registro litológico obtenido, se pueden relacionar las partes inferiores de los pozos con las unidades distales de los centros volcánicos referidos. De la misma forma, en Tepoztlán y Malinalco (estados de Morelos y de México), al sur de la CM, se encuentran expuestos los primeros episodios volcánicos de la FVTM, con edades que varían entre los $\sim 22.8$ y $18.8 \mathrm{Ma}$, para los cuales se ha sugerido en trabajos previos una correlación temporal con las edades reportadas en varios pozos profundos de la $\mathrm{CM}$.

A partir de la información geológica disponible, se argumenta que el proceso de subsidencia observado en la CM es un rasgo tectónico contemporáneo a la FVTM, que no se encuentra relacionado a la tectónica del Paleógeno de la región. La ausencia de espesores significativos (no superiores a $1000 \mathrm{~m}$ ) de rocas volcánicas o sedimentarias cuya edad probable es del Paleógeno entre las sucesiones del Neógeno y las capas marinas del Cretácico en el subsuelo de la CM sugiere que la subsidencia ocurrió principalmente durante el Neógeno.
\end{abstract}

Palabras clave: Cuenca de México, Faja Volcánica Trans-Mexicana, Sierra Madre del Sur, registros de pozos.

\begin{abstract}
In this work we review the possible correlation between the deep subsoil units of the Mexico Basin (MB), that are known through the lower successions cut by wells, and the Cenozoic stratigraphic record previous to the Trans-Mexican Volcanic Belt (TMVB), which is exposed in the northern part of the Sierra Madre del Sur (SMS). South of the MB, recent stratigraphic studies provide new geochronological data from some eruptive centers of the northern-central sector of the SMS, such as the Taxco and Huautla volcanic fields, and the Tilzapotla caldera. These volcanic fields represent the last volcanic record of the Paleocene SMS arc that developed before the inception of the TMVB. Based on lithological analogies, the stratigraphic position and the thickness of the obtained lithological
\end{abstract}


record, the lower parts of the deep wells drilled in the MB can be related to the distal units of the previously mentioned volcanic centers. In the same way, in Tepoztlán and Malinalco (states of Morelos and México), located south of the MB, the first volcanic episodes of the TMVB are exposed, with ages ranging from $\sim 22.8$ and $18.8 \mathrm{Ma}$, that have been correlated by other authors to the ages reported in various deep wells of the $M B$.

Based on the available geologic information, we consider that the process of subsidence observed in the MB is contemporaneous with the TMVB, and that it is not related to the Paleogene tectonics of the region. The absence of significant volumes of volcanic or sedimentary rocks of probable Paleogene age (generally less than $1000 \mathrm{~m}$ thick) between the Neogene successions and the Cretaceous marines units in the MB subsoil, strongly suggests that subsidence mainly took place during the Neogene.

Keywords: Mexico Basin, Trans-Mexican Volcanic Belt, Sierra Madre del Sur, well records.

\section{Introducción}

La Cuenca de México (CM) es una entidad geomorfológica endorreica que se ubica en la porción centro-oriental de la Faja Volcánica Trans-Mexicana (FVTM) (Gómez-Tuena et al., 2005; Ferrari et al., 2012) (Figura 1); tiene una superficie de aproximadamente 9540 $\mathrm{km}^{2}$, y la planicie central presenta una altura promedio de 2240 msnm (Lugo-Hubp et al., 1994; de Cserna et al., 1988; Enciso-De la Vega, 1992). La CM incluye una extensa planicie lacustre que está delimitada por cuatro sierras de origen volcánico: la Sierra Chichinautzin al sur, la Sierra de las Cruces al poniente, la Sierra Nevada al oriente, y la Sierra de Pachuca y Tezontlalpan al norte (de Cserna et al., 1988; Enciso-De la Vega, 1992; García-Palomo et al., 2002, 2008; Zamorano-Orozco et al., 2002; Siebe et al., 2004a; Macías et al., 2012) (Figura 1). Además, alberga algunos conjuntos volcánicos de diversas dimensiones, como las sierras de Guadalupe y de Santa Catarina (de Cserna et al., 1988; Lugo-Hubp et al., 1994; Zamorano-Orozco et al., 2002; García-Palomo et al., 2008) (Figura 2).

Se considera que la $\mathrm{CM}$ es una depresión profunda que se originó en repuesta a la actividad volcánica y tectónica de la región, y que los materiales que la rellenan son dominantemente volcánicos (Siebe et al., 2004a; GarcíaPalomo et al., 2008). En forma general, el relleno de la CM está conformado por depósitos lacustres en la parte superior, y derrames de lava, materiales epiclásticos y depósitos piroclásticos en la parte inferior (Pérez-Cruz, 1988; Lozano-García y Ortega-Guerrero, 1998; GarcíaPalomo et al., 2008; Arce et al., 2013a).

La CM representa una región de importancia por varias razones, entre las cuales destaca que en el sector suroccidental se edifica la Ciudad de México y sus zonas conurbadas. Si bien la zona urbana tiene un área aproximada de $\sim 1500 \mathrm{~km}^{2}$ que sólo representa el $\sim 16 \%$ de la superficie total de la CM (Figura 2), la región alberga una población de alrededor de 21.4 millones de personas (INEGI, 2013), una de las mayores concentraciones de habitantes del planeta. Una metrópoli de tal magnitud invariablemente está vinculada a múltiples problemáticas, a menudo asociadas con factores de tipo geológico: por ejemplo, la $\mathrm{CM}-\mathrm{y}$ por ende la Ciudad de México - muestra una alta tasa de subsidencia, la cual excede los $350 \mathrm{~mm} /$ año en promedio en las zonas críticas; a este proceso se han vinculado procesos de fracturamiento y fallamiento (Santoyo-Villa et al., 2005; Cabral-Cano et al., 2008). Lo anterior hace necesario conocer con más detalle las características geológicas y estructurales de la CM, la naturaleza del subsuelo profundo, el tipo de procesos que le dieron origen, y los vínculos entre la $\mathrm{CM}$ y la geología expuesta en las regiones circundantes.

La perforación del Pozo Texcoco en la porción noreste de la Ciudad de México (Proyecto Texcoco, 1966 - 1969) permitió conocer por primera vez una columna estratigráfica continua de la CM hasta una profundidad de $2065 \mathrm{~m}$, cerca de donde se cortaron depósitos de un conglomerado calcáreo y capas de anhidrita (Hiriart y Graue, 1969; Oviedo de León, 1970) (Figura 3). A raíz del terremoto de 1985 que afectó severamente la Ciudad de México, se realizaron diversos estudios geológicos y geofísicos, y se perforaron cuatro pozos profundos, que proporcionaron nueva información sobre la estratigrafía, geocronología y estructura del subsuelo de la CM (Figura 3); esta información sirvió para replantear algunas de las hipótesis sobre su origen (de Cserna et al., 1988; Pérez-Cruz, 1988; Vázquez-Sánchez y Jaimes-Palomera, 1989; Enciso-De la Vega, 1992; Chávez-Pérez, 1993; Mooser et al., 1996). La perforación del Pozo de San Lorenzo Tezonco, realizada recientemente por el Sistema de Aguas al oriente de la Ciudad de México, permitió conocer más detalles sobre la estratigrafía, ampliar la información geocronológica sobre las unidades perforadas por el método ${ }^{40} \mathrm{Ar} /{ }^{39} \mathrm{Ar}$, y obtener datos geoquímicos de las rocas volcánicas que conforman el subsuelo profundo de la CM (Arce et al., 2013a, 2015) (Figura 3). Asimismo, en los últimos años se han realizado estudios geológicos-vulcanológicos en la porción meridional de la CM, que proporcionan un conocimiento más detallado de la litología y edad de los primeros episodios volcánicos de la FVTM (García-Palomo et al., 2000; Lenhardt et al., 2010); y estudios estratigráficos-geocronológicos de los últimos episodios del volcanismo cenozoico previo a la FVTM, expuestos en la porción norte-central de la SMS (Morán-Zenteno et al., 2004, 2007; González-Torres et al., 2013) (Figura 4). 


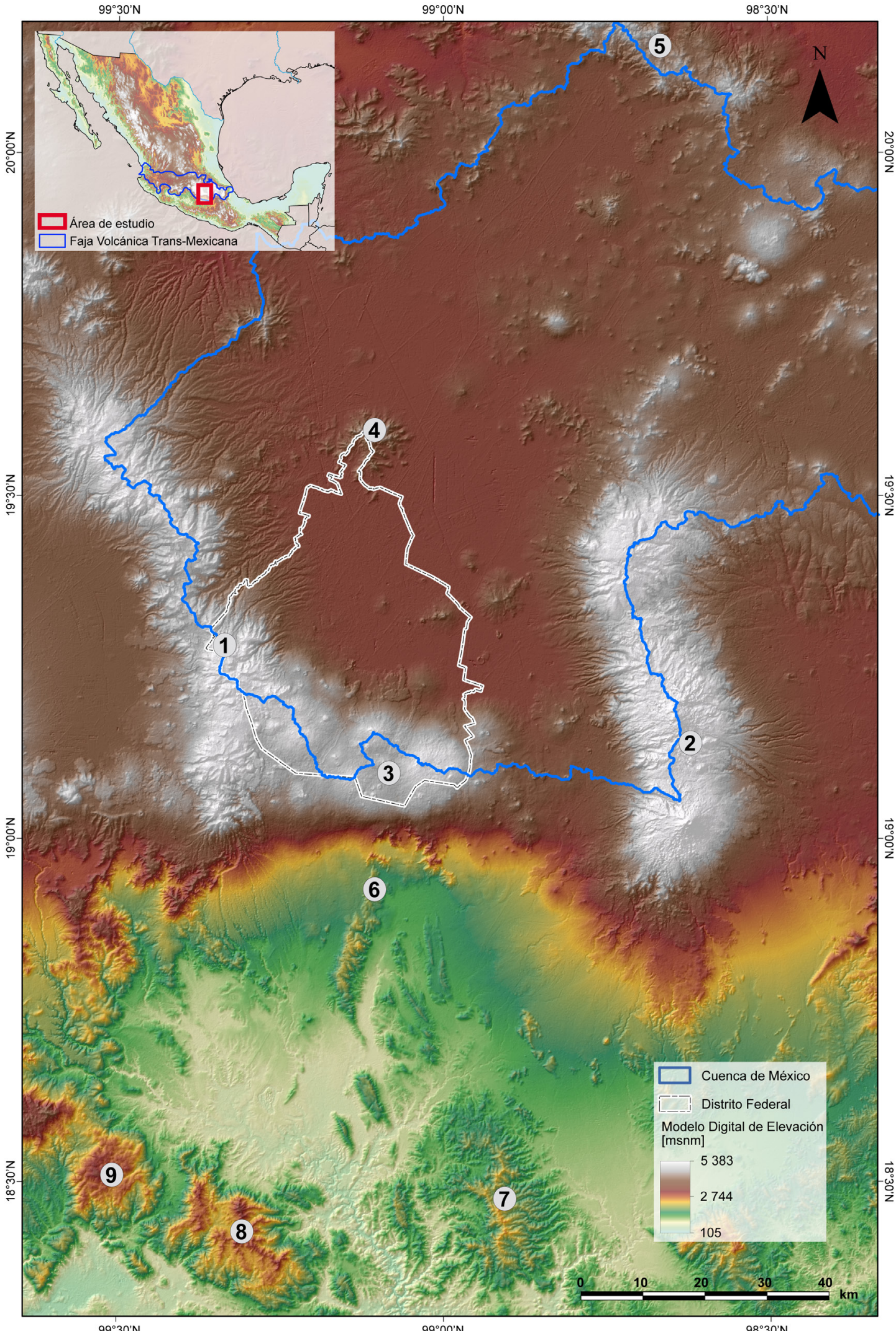

Figura 1. Modelo de elevación digital que muestra la localización de la zona de estudio en el que se indican los contornos del sector sur de la Cuenca de México y de la ciudad de México, así como las sierras que limitan a estas últimas: (1) Sierra de las Cruces, (2) Sierra Nevada, (3) Sierra de Chichinautzin; (4) Sierra de Guadalupe; (5) Sierra de Pachuca. En la porción sur del mapa se muestran la región de (6) Tepoztlán, Morelos, en donde se encuentran expuestos los primeros episodios volcánicos de la Faja Volcánica Trans-Mexicana en su sector centro-oriental y los campos volcánicos de la Sierra Madre del Sur: (7) Huautla, Morelos, (8) la caldera de Tilzapotla y (9) Taxco, Guerrero. 


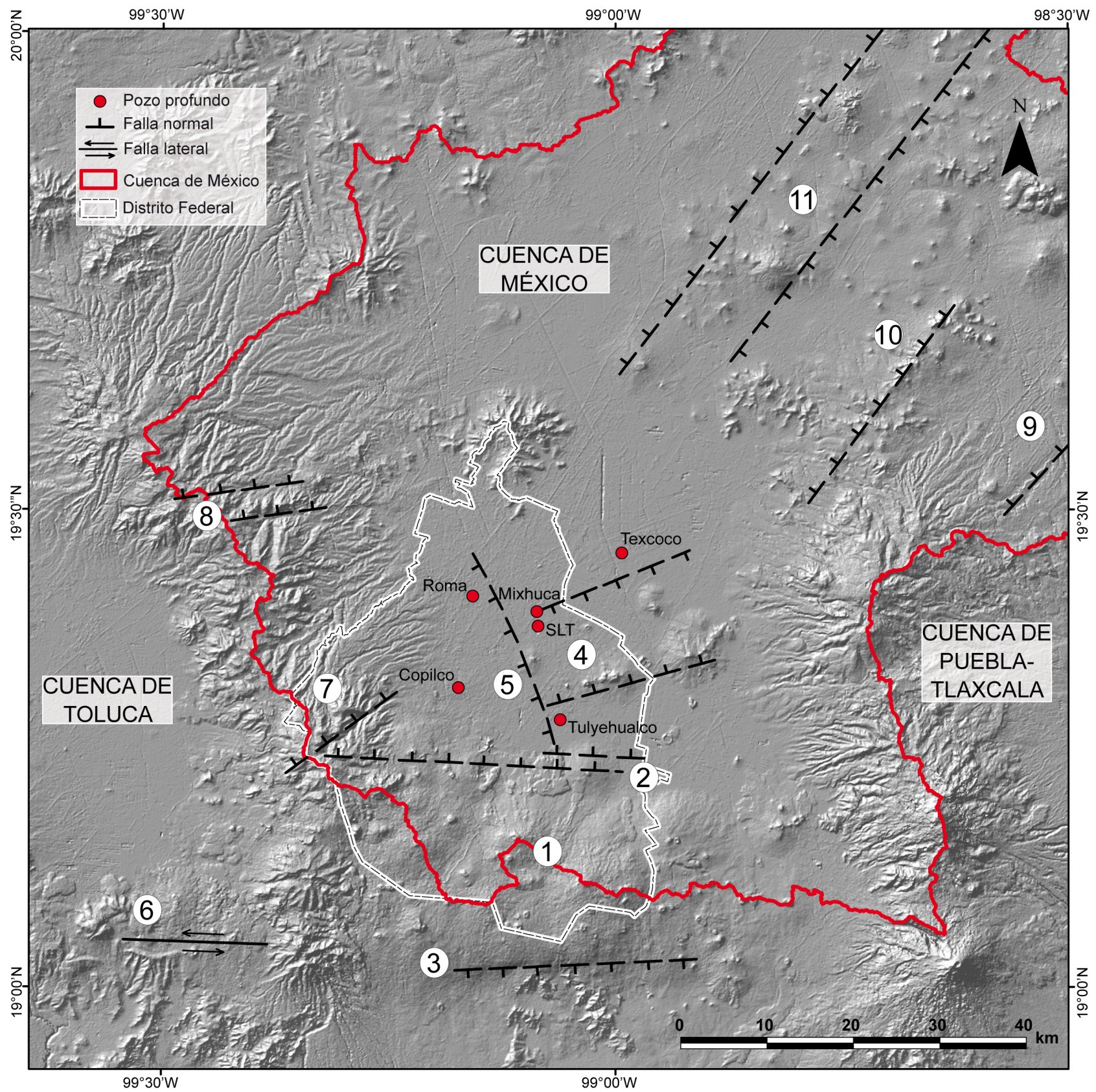

Figura 2. Modelo de elevación de la Cuenca de México en la que se indican con círculos rojos la ubicación y nombre de los seis pozos profundos perforados en la Cuenca de México: Copilco-1; Roma-1; Tulyehualco-1; Mixhuca-1; Texcoco-1 y SLT (San Lorenzo Tezonco). Se muestran algunas de las estructuras geológicas citadas como son: (1) Pilar (horst) de Chichinautzin (Siebe et al., 2004b), (2) Sistema de fallas de Xochimilco y Xicomulco (García-Palomo et al., 2008), (3) sistema de Fallas de la Pera (Delgado-Granados et al., 1995; Alaniz-Álvarez y Nieto-Samaniego, 2005), (4) Graben de Santa Catarina (Arce et al., 2013a) (5) Falla Mixhuca (Pérez-Cruz, 1988), (6) Sistema de Fallas Tenango (Norini et al., 2006), (7) Falla Contreras (Arce et al., 2015), (8) Sistema de Fallas de Ixtlahuaca y Otomí (García-Palomo et al., 2008), (9) Segmento sur del Sistema de Fallas Apan-Tláloc, (10) Falla Texcoco y 11) Sistema de fallas Tizayuca (García-Palomo et al., 2002).

Toda esta información ofrece la oportunidad de revisar la posible correlación entre el subsuelo profundo de la CM y las unidades litológicas expuestas en la porción norte de la SMS; y de discutir los modelos propuestos sobre la temporalidad y el contexto tectónico en el que se desarrolló la cuenca.

\section{Marco geológico de la CM y su entorno}

Algunas de las características de las sierras de origen volcánico que delimitan a la CM son las siguientes: la Sierra de las Cruces (límite occidental) tiene una longitud de 110 $\mathrm{km}$, y un ancho variable entre $47 \mathrm{~km}$ en su parte norte y 27 

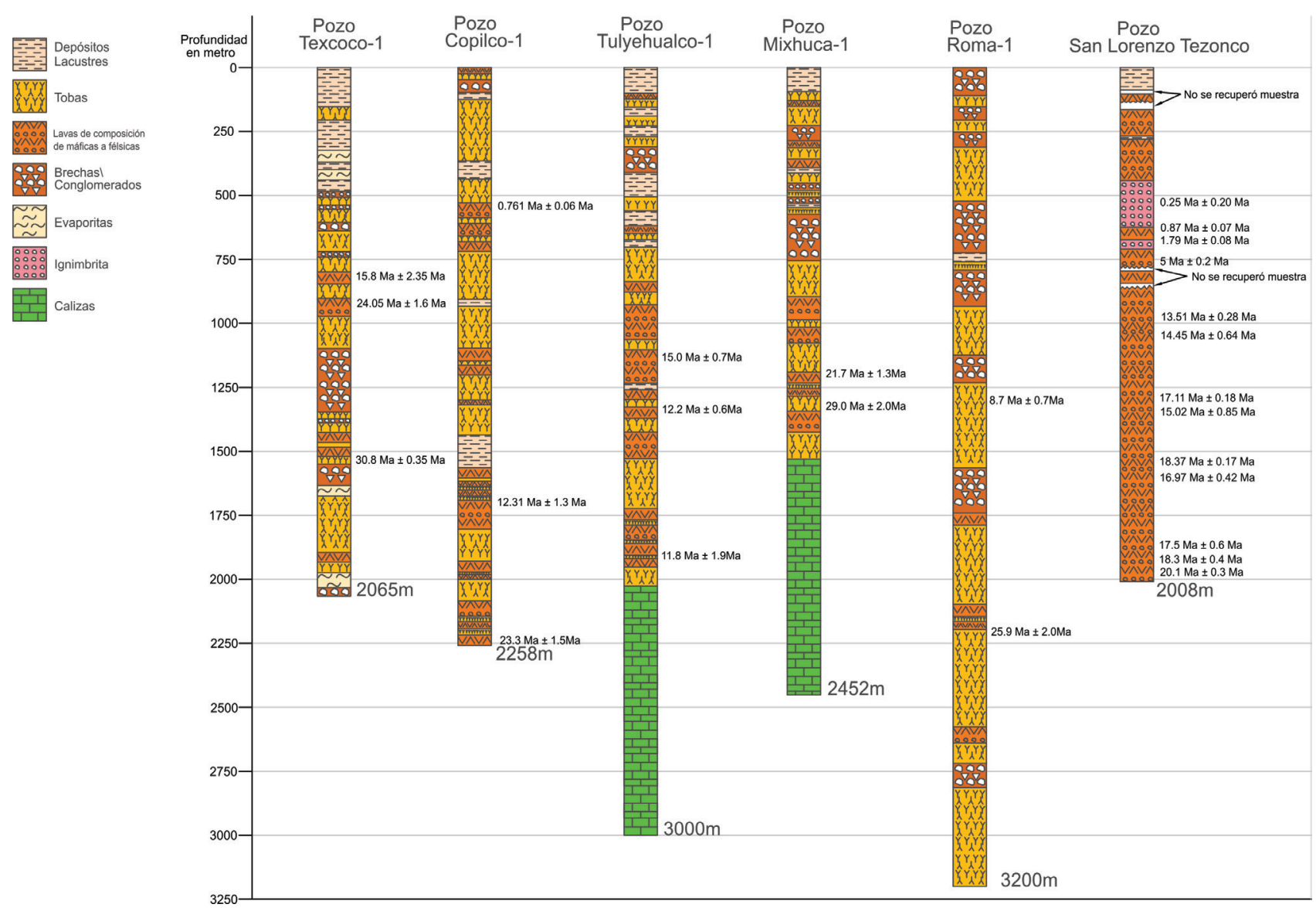

Figura 3. Columnas estratigráficas de los de los pozos profundos perforados en la Cuenca de México, en donde se muestran las edades obtenidas para cada uno de ellos. La litología del pozo de San Lorenzo Tezonco se simplificó para homologar la litología reportada en los otros pozos (Oviedo de León, 1970; Pérez-Cruz, 1988 y Arce et al., 2013a, 2105).

km en su parte sur (García-Palomo et al., 2008; Arce et al., 2008); sus edades varían entre 3.7 y $0.7 \mathrm{Ma}$, por lo que es la sierra más antigua en la $\mathrm{CM}$. En el campo volcánico Chichinautzin (límite meridional), las edades más antiguas varían entre 1.2 y 0.9 Ma en su porción oeste y sur, y son menores de 0.04 Ma en su mayor parte (Siebe et al., 2004a; Arce et al., 2013b). La Sierra Nevada (límite oriental) tiene una longitud de $45 \mathrm{~km}$ y una altura máxima de $5454 \mathrm{~m}$ en el volcán Popocatépetl (Macías et al., 2012); sus edades varían de 1.8 Ma al actual (Macías et al., 2012) (Figura 1).

Asociados a las cadenas montañosas de origen volcánico que limitan la $\mathrm{CM}$, existen sistemas de fallas que muestran orientaciones preferenciales E-W y NE-SW (Figura 2). El sector sur de la CM está limitado por los sistemas de fallas normales Xochimilco y Xicomulco, con orientación E-W, cuyo bloque caído está al norte (García-Palomo et al., 2008). Por su alineación, se ha sugerido que dichas fallas se conectan hacia el poniente con el sistema de fallas Tenango (Norini et al., 2006). Al sur del sistema Xochimilco, de forma aproximadamente paralela se ubica el sistema de fallas normales de La Pera, cuyo bloque de techo ubicado hacia Cuernavaca, Morelos (Delgado-Granados et al., 1995; Alaniz-Álvarez y Nieto-Samaniego, 2005) define el pilar tectónico de Chichinautzin (Siebe et al., 2004b; Colín-Rodríguez, 2006) (Figura 2). La Sierra de las Cruces está constituida por tres bloques delimitados por fallas con dirección aproximada E-W: las fallas de Ixtlahuaca y Otomí limitan el bloque norte y central, la falla Contreras con dirección NE-SW forma parte del bloque central, mientras que la falla Xochimilco podría delimitar el bloque meridional (García-Palomo et al., 2008) (Figura 2). El lineamiento N-S que define la Sierra de las Cruces podría formar parte del sistema de fallas regionales Taxco-Querétaro; de hecho, aunque muestra una dirección dominante NW-SE, a este sistema se asocian fallas con orientación N-S, como la de Perales al sur de Querétaro (Alaniz-Álvarez et al., 2002; Alaniz-Álvarez y Nieto-Samaniego, 2005; Norini et al., 2006). Desde un punto de vista estructural, el límite norte de la CM está definido por un sistema de fallas con orientación NE-SW, que incluyen las fallas de Tizayuca y Texcoco (García-Palomo et al., 2002), y la falla Apan-Tláloc (Campos-Enríquez et al., 2003) (Figura 2).

En la zona interna de la CM se ha documentado una falla normal con dirección preferencial NW-SE que cruza la Ciudad de México, a la que se ha denominado Falla de Mixhuca, cuyo bloque caído corresponde al segmento 


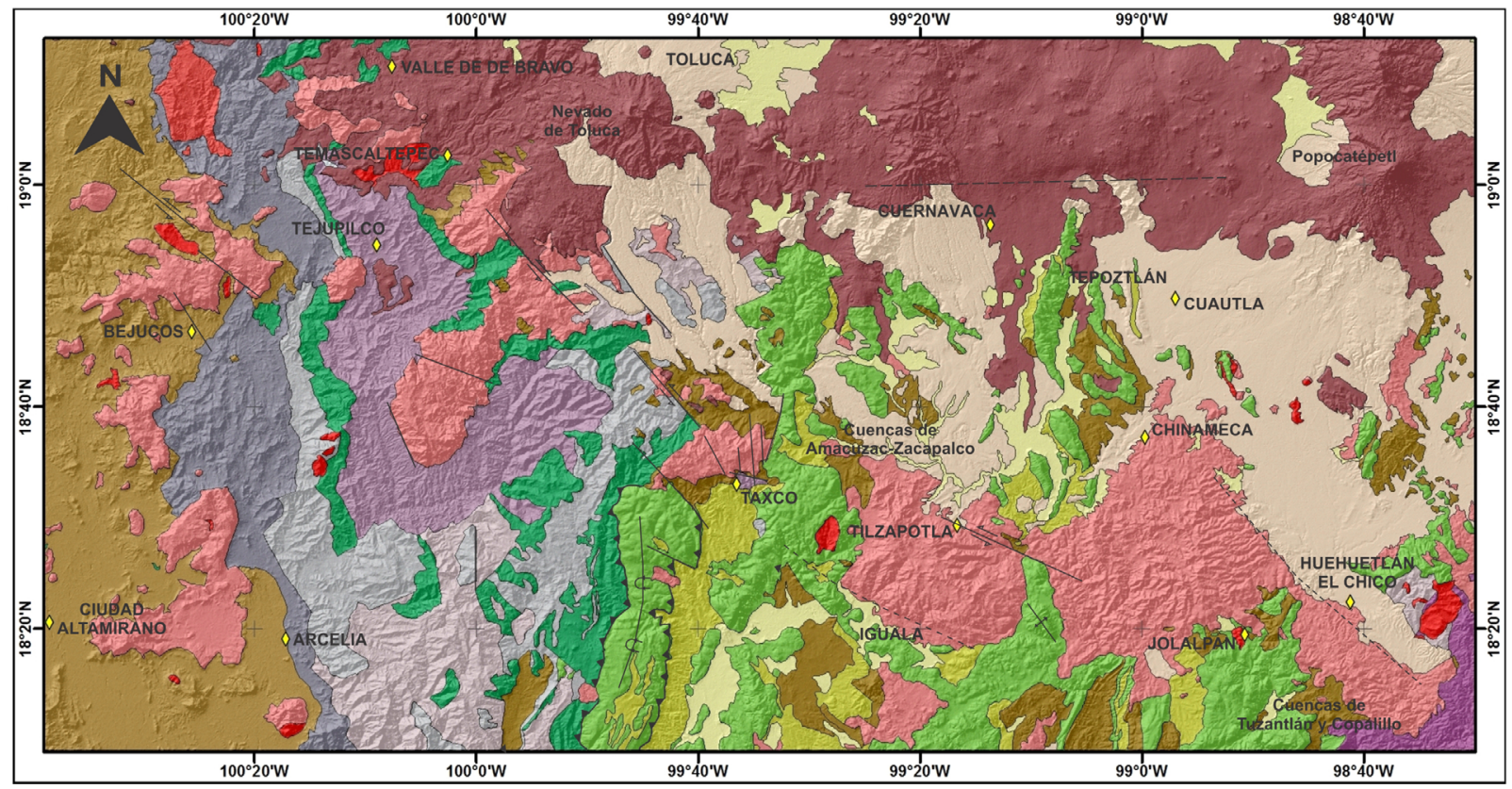

0 $20 \mathrm{~km}$

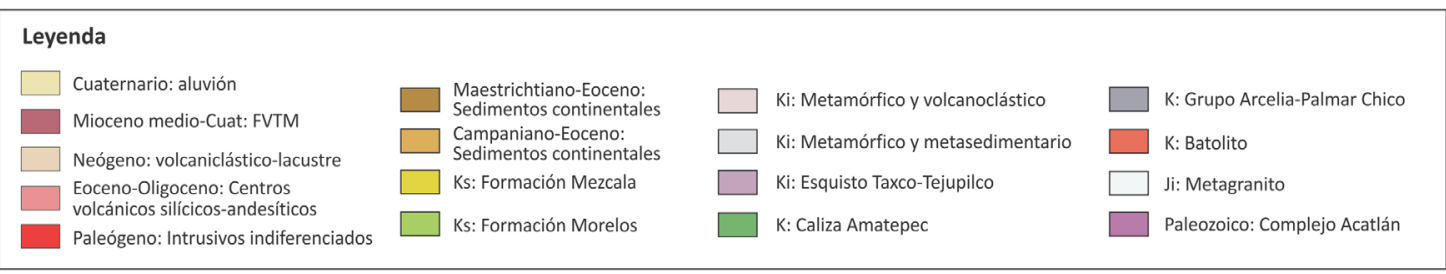

Figura 4. Mapa geológico simplificado del sector norte-central de la Sierra Madre del Sur que muestra la ubicación de los centros volcánicos anteriores a la FVTM ubicados al sur de la Cuenca de México, en los estados de Guerrero y Morelos, como son el campo volcánico de Huautla, la caldera de Tilzapotla y de Taxco, así como la región de Tepoztlán, área en donde aflora el registro más antiguo de la FVTM en el sector centro oriental.

poniente (Pérez-Cruz, 1988) (Figura 2); y un sistema de fallas y lineamientos regionales con orientación NE-SW denominado zona de cizallamiento Tenochtitlan (de Cserna et al., 1988; Aguayo-Camargo et al., 1989; Santoyo-Villa et al., 2005), del que forma parte el graben de Santa Catarina (Arce et al., 2013a). El entorno estructural de la $\mathrm{CM}$, así como los pozos profundos perforados, permiten inferir que la CM está constituida por un amplio sistema de pilares y bloques escalonados, que definen la base de la depresión como una superficie irregular; el basamento está constituido por secuencias sedimentarias del Mesozoico sepultadas a profundidades variables entre 1600 y más de $3200 \mathrm{~m}$, como indican los datos provenientes de los pozos Mixhuca-1 y Roma-1 (Pérez-Cruz, 1988; Aguayo-Camargo et al., 1989; García-Palomo et al., 2008). El contexto anterior, aunado a los conglomerados y brechas cortados por los pozos, sugieren que el relleno más antiguo de la CM está constituido por depósitos continentales de cuencas intermontanas del Cenozoico, con espesor no mayor a los $100 \mathrm{~m}$, que sobreyacen a las secuencias marinas cretácicas
(Oviedo de León, 1970; Pérez-Cruz, 1988) (Figura 3).

Los sistemas de fallas descritos no coinciden con las fallas laterales izquierdas de orientación NW que caracterizan la deformación tectónica contemporánea al volcanismo paleógeno de la parte norte de la SMS (Figura 4), lo que sugiere un escenario dinámico diferente entre el Paleógeno y el Neógeno en la región.

\section{El registro estratigráfico del subsuelo de la $\mathrm{CM}$}

La estratigrafía del subsuelo de la $\mathrm{CM}$ se ha integrado a partir de la información de varios pozos profundos y ha sido descrita en diferentes estudios (de Cserna et al., 1988; PérezCruz, 1988; Enciso-De la Vega, 1992; Arce et al., 2013a, 2015). La base está constituida por la Formación Morelos del Cretácico inferior, y por depósitos de conglomerados calcáreos y anhidritas similares al Grupo Balsas. El relleno está formado por depósitos lacustres en la cima, y productos volcánicos. Estos últimos incluyen derrames de lavas de 
composición variable desde basalto a dacita, con dominancia de andesitas; depósitos piroclásticos de tobas e ignimbritas; y una amplia variedad de materiales volcaniclásticos con edades que varían desde el Oligoceno hasta el Cuaternario (Schlaepfer, 1968; Mooser, 1975; de Cserna et al., 1988; Vázquez-Sánchez y Jaimes-Palomera, 1989; Enciso-De la Vega, 1992; Pérez-Cruz, 1998; Arce et al., 2013a) (Figura 3).

Los seis pozos profundos perforados en la CM cubren un rango de profundidad que alcanza desde 2008 a $3200 \mathrm{~m}$ (Oviedo de León, 1970; PEMEX, 1987a, b, c en Pérez-Cruz, 1988; Arce et al., 2013a, 2015). El mayor espesor litológico perforado en la CM (3200 m) se obtuvo a partir del pozo Roma-1, que sin embargo no logró alcanzar el basamento de calizas; en cambio, el pozo Mixhuca-1 corta las calizas a $\sim 1600 \mathrm{~m}$ de profundidad (Figura 3 ). Se han realizado fechamientos isotópicos en todos ellos (Tabla 1) (SHCP, 1969; Pérez-Cruz, 1988; Arce et al., 2013a, 2015). Existe un reporte posterior de fechamientos ${ }^{39} \mathrm{Ar} /{ }^{40} \mathrm{Ar}$ realizados en los núcleos de los pozos Copilco-1, Tulyehualco-1 y Mixhuca-1 (Ferrari et al., 2003), en el cual se señala que las edades obtenidas difieren de los fechamientos previos; sin embargo, el reporte corresponde a un resumen y no incluye las profundidades de las muestras analizadas. Los trabajos realizados sobre la geocronología de las sierras volcánicas que limitan a la CM (Arce et al., 2013a, 2013b, 2015 y referencias citadas) han permitido inferir la procedencia de varios de los productos volcánicos que rellenan la porción superior de la CM.

De acuerdo a Pérez-Cruz (1988), el relleno de la cuenca está conformado por cuatro unidades, que de la base a la cima son: a) calizas del Cretácico que constituyen el basamento; b) Unidad Volcánica Inferior (UVI), constituida por productos volcánicos efusivos y piroclásticos; c) Unidad Volcánica Superior (UVS), constituida esencialmente por rocas piroclásticas, con una menor proporción de rocas efusivas; d) unidad de depósitos lacustres y fluviales representados por arcillas, limos y arenas. Pérez-Cruz (1988) correlaciona la UVI con la provincia ignimbrítica de la Sierra Madre Occidental; mientras que la UVS corresponde a las rocas de la FVTM.

\section{Estratigrafía de la región norte de Morelos y Guerrero}

Las sucesiones volcánicas presentes al norte de los estados de Morelos y Guerrero descansan sobre capas de calizas plegadas de la Formación Morelos, evaporitas de la Formación Huitzuco del Cretácico, y sobre el Grupo Balsas, conformado por conglomerados, areniscas, limolitas y material volcánico intercalado, con un rango de edad Paleoceno - Eoceno (Fries, 1960).

En el área de Amacuzac-Zacapalco los conglomerados del Grupo Balsas contienen algunos derrames intercalados de andesita y basalto, así como de tobas denominadas Formación Tepetlapa (Morán-Zenteno et al., 2007). Para uno de los derrames se reporta una edad de $44.5 \pm 0.7 \mathrm{Ma}$, en tanto que para las tobas se obtuvieron edades de $38.5 \pm$ 0.7 y $35.1 \pm 0.4$ (Morán-Zenteno et al., 2007) (Figura 5).

El Grupo Balsas subyace al registro volcánico del Cenozoico pre-FVTM en esta región, a través de un contacto transicional. Las sucesiones volcánicas están relacionadas con la actividad magmática del Eoceno-Oligoceno de la caldera de Tilzapotla y de los campos volcánicos de Taxco y Huautla. Consisten principalmente de ignimbritas silícicas y derrames lávicos con composición variable de andesita basáltica a dacita, los cuales presentan un rango de edades entre $\sim 38$ y 28 Ma (Alaniz-Álvarez et al., 2002; MoránZenteno et al., 2004; González-Torres et al., 2013).

La litología y alcance estratigráfico de las unidades en los diferentes centros volcánicos aparecen sintetizados en la Figura 5. Posteriormente a los depósitos continentales del Grupo Balsas, la instauración de la actividad volcánica fue gradual; inicialmente se emitieron depósitos piroclásticos de volumen reducido, a los cuales siguió una voluminosa actividad silícica representada por varios pulsos de ignimbritas; de estos pulsos, el más joven está representado por la Ignimbrita Maravillas del campo volcánico de Huautla, cuya edad es diferente a la de la Ignimbrita Tilzapotla (González-Torres et al., 2013). En el campo volcánico de Huautla, la actividad volcánica declinó en un tiempo diferente respecto a los otros centros volcánicos de la región; en dicho campo, los últimos eventos se caracterizan por la emisión de lavas de composición variable desde andesita basáltica a dacita, con una mayor abundancia de andesitas, que corresponden a las andesitas de Tepalcingo y Chimalacatlán, cuyas edades más jóvenes son de aproximadamente $28 \mathrm{Ma}$ (González-Torres et al., 2013).

En la región de Malinalco y Tepoztlán, respectivamente al sur del estado de México y al norte del estado de Morelos, se ubica una franja de transición entre la CM y la SMS, en donde aflora un conjunto denominado Formación Tepoztlán (Figuras 1 y 4), integrado por sucesiones de derrames de lavas intercaladas en depósitos fluviales, depósitos piroclásticos, y productos volcaniclásticos y fluviales. Para esta formación se han reportado edades K/Ar de $21.86 \pm$ 0.2 a $19.4 \pm 1.2 \mathrm{Ma}$, por lo que dichos productos se han asociado a los primeros eventos magmáticos de la FVTM del Mioceno inferior (García-Palomo et al., 2002; Lenhardt et al., 2010).

El espesor máximo alcanzado por la Formación Tepoztlán es inferior a $800 \mathrm{~m}$ y se ha agrupado en tres subdivisiones establecidas de acuerdo al proceso dominante de su depósito: Miembro Malinalco dominado por procesos fluviales; Miembro San Andrés constituido principalmente por productos volcánicos como lavas y depósitos piroclásticos; Miembro Tepozteco con litología dominante asociado a depósitos de flujos en masa tipo lahares (García-Palomo et al., 2002; Lenhardt et al., 2010) (Figura 5).

En la integración de una columna compuesta por la actividad volcánica anterior a la FVTM y la de la Formación 
Tabla 1. Edades radiométricas de los pozos profundos de la Cuenca de México y de zonas adyacentes.

\begin{tabular}{|c|c|c|c|c|c|c|c|c|c|}
\hline $\begin{array}{l}\text { Muestra/prof. } \\
\text { (m) }\end{array}$ & Localidad /Pozo & Long (W) & Lat $(\mathbf{N})$ & Unidad & Roca & $\begin{array}{l}\text { Material } \\
\text { fechado }\end{array}$ & Edad (Ma) & Método & Referencia \\
\hline \multicolumn{10}{|c|}{ Afloramientos sector sur-central de la FVTM y sector norte-central de la SMS } \\
\hline BI & San Nicolás & $99^{\circ} 30^{\prime} 00^{\prime \prime}$ & $18^{\circ} 58^{\prime} 30^{\prime \prime}$ & And. Bas. San Nicolás & $\mathrm{AB}$ & RT & $21.6 \pm 1.0$ & $\mathrm{~K}-\mathrm{Ar}$ & 5,6 \\
\hline BMS3 & Tenango & $99^{\circ} 37^{\prime} 20^{\prime \prime}$ & $19^{\circ} 05^{\prime} 15^{\prime \prime}$ & Secuencia Máfica Basal & A & RT & $7.5 \pm 0.4$ & $\mathrm{~K}-\mathrm{Ar}$ & 5,6 \\
\hline Нu-242 & $\mathrm{CVH}$ & $99^{\circ} 02^{\prime} 30^{\prime \prime}$ & $18^{\circ} 27^{\prime} 43^{\prime \prime}$ & Andesita Chimalacatlán & A & RT & $28.2 \pm 1.1$ & $\mathrm{~K}-\mathrm{Ar}$ & 9 \\
\hline Hu-190305 & $\mathrm{CVH}$ & $98^{\circ} 06^{\prime} 18^{\prime \prime}$ & $18^{\circ} 26^{\prime} 42^{\prime \prime}$ & Andesita Chimalacatlán & A & RT & $29.7 \pm 1.4$ & $\mathrm{~K}-\mathrm{Ar}$ & 9 \\
\hline $\mathrm{Hu}-384$ & $\mathrm{CVH}$ & $98^{\circ} 53^{\prime} 48^{\prime \prime}$ & $18^{\circ} 36^{\prime} 23^{\prime \prime}$ & Andesita Tepalcingo & A & RT & $28.1 \pm 1.0$ & $\mathrm{~K}-\mathrm{Ar}$ & 9 \\
\hline \multicolumn{10}{|c|}{ Formación Tepoztlán } \\
\hline M 210 & al SE de Malinalco & $99^{\circ} 29^{\prime} 05.91 "$ & $18^{\circ} 55^{\prime} 51.60^{\prime \prime}$ & Formación Tepoztlán & D-A & RT & $20.9 \pm 3.1$ & $\mathrm{~K}-\mathrm{Ar}$ & 7,12 \\
\hline TE 5 & al $\mathrm{N}$ de Tepoztlán & $99.10^{\circ}$ & $18.99^{\circ}$ & Formación Tepoztlán & D-A & RT & $19.4 \pm 1.2$ & $\mathrm{~K}-\mathrm{Ar}$ & 7 \\
\hline SO14 & al SW de Tlayacapan & $98.98^{\circ}$ & $18.94^{\circ}$ & Formación Tepoztlán & D-A & RT & $24.1 \pm 3.6$ & $\mathrm{~K}-\mathrm{Ar}$ & 7 \\
\hline TL $250-5 p$ & al SW de Tlayacapan & $98.59^{\circ}$ & $18.56^{\circ}$ & Formación Tepoztlán & D-A & RT & $20.0 \pm 1.0$ & $\mathrm{~K}-\mathrm{Ar}$ & 7 \\
\hline TL 233-12p & al NE de Tlayacapan & $98.96^{\circ}$ & $18.99^{\circ}$ & Formación Tepoztlán & D-A & RT & $19.8 \pm 0.8$ & $\mathrm{~K}-\mathrm{Ar}$ & 7 \\
\hline SAC & cerca de San Andrés & $99^{\circ} 06^{\prime} 44.19^{\prime \prime}$ & $18^{\circ} 58^{\prime} 11.85^{\prime \prime}$ & Formación Tepoztlán & D-A & RT & $21.86 \pm 0.20$ & $\mathrm{Ar}-\mathrm{Ar}$ & 7,12 \\
\hline DY1 & cerca de Malinalco & $99^{\circ} 29^{\prime} 15.08^{\prime \prime}$ & $18^{\circ} 55^{\prime} 54.93^{\prime \prime}$ & dique emplazado en FT & Di & RT & $15.83 \pm 1.31$ & $\mathrm{Ar}-\mathrm{Ar}$ & 7,12 \\
\hline \multicolumn{10}{|c|}{ Pozos profundos de la Cuenca de México } \\
\hline $512-514$ & SLT & $99^{\circ} 05^{\prime} 5.25^{\prime \prime}$ & $19^{\circ} 22^{\prime} 44.27^{\prime \prime}$ & Sierra de Chichinautzin ${ }^{10}$ & A & $\mathrm{MC}$ & $0.25 \pm 0.20^{\mathrm{c}}$ & Ar-Ar & 8 \\
\hline 662-664 grupo 1 & SLT & $99^{\circ} 05^{\prime} 5.25^{\prime \prime}$ & $19^{\circ} 22^{\prime} 44.27^{\prime \prime}$ & Sierra las Cruces ${ }^{10}$ & $\mathrm{D}$ & $\mathrm{MC}$ & $0.86 \pm 0.07^{c}$ & Ar-Ar & 8 \\
\hline 662-664 grupo 2 & SLT & $99^{\circ} 05^{\prime} 5.25^{\prime \prime}$ & $19^{\circ} 22^{\prime} 44.27^{\prime \prime}$ & Sierra las Cruces ${ }^{10}$ & $\mathrm{D}$ & $\mathrm{MC}$ & $1.79 \pm 0.08^{c}$ & Ar-Ar & 8 \\
\hline $854-856$ & SLT & $99^{\circ} 05^{\prime} 5.25^{\prime \prime}$ & $19^{\circ} 22^{\prime} 44.27^{\prime \prime}$ & Sierra las Cruces ${ }^{10}$ & IR & $\mathrm{zr}$ & $5+0.20 /-0.80$ & $\mathrm{U}-\mathrm{Pb}$ & 8 \\
\hline $964-966$ & SLT & $99^{\circ} 05^{\prime} 5.25^{\prime \prime}$ & $19^{\circ} 22^{\prime} 44.27^{\prime \prime}$ & Volcanismo miocénico & A & $\mathrm{MC}$ & $13.51 \pm 0.28^{c}$ & Ar-Ar & 8 \\
\hline 1190 & SLT & $99^{\circ} 05^{\prime} 5.25^{\prime \prime}$ & $19^{\circ} 22^{\prime} 44.27^{\prime \prime}$ & Volcanismo miocénico & A & $\mathrm{MC}$ & $14.45 \pm 0.64^{\mathrm{c}}$ & $\mathrm{Ar}-\mathrm{Ar}$ & 8 \\
\hline 1286 & SLT & $99^{\circ} 05^{\prime} 5.25^{\prime \prime}$ & $19^{\circ} 22^{\prime} 44.27^{\prime \prime}$ & Volcanismo miocénico & A & $\mathrm{MC}$ & $17.11 \pm 0.18^{c}$ & Ar-Ar & 8 \\
\hline 1408 & SLT & $99^{\circ} 05^{\prime} 5.25^{\prime \prime}$ & $19^{\circ} 22^{\prime} 44.27^{\prime \prime}$ & Volcanismo miocénico & A & $\mathrm{MC}$ & $15.02 \pm 0.85^{\mathrm{c}}$ & $\mathrm{Ar}-\mathrm{Ar}$ & 8 \\
\hline 1550 & SLT & $99^{\circ} 05^{\prime} 5.25^{\prime \prime}$ & $19^{\circ} 22^{\prime} 44.27^{\prime \prime}$ & Formación Tepoztlán & A & $\mathrm{MC}$ & $18.37 \pm 0.17^{c}$ & $\mathrm{Ar}-\mathrm{Ar}$ & 8 \\
\hline 1654 & SLT & $99^{\circ} 05^{\prime} 5.25^{\prime \prime}$ & $19^{\circ} 22^{\prime} 44.27^{\prime \prime}$ & Formación Tepoztlán & A & $\mathrm{MC}$ & $16.97 \pm 0.42^{\mathrm{c}}$ & Ar-Ar & 8 \\
\hline 1806 & SLT & $99^{\circ} 05^{\prime} 5.25^{\prime \prime}$ & $19^{\circ} 22^{\prime} 44.27^{\prime \prime}$ & Formación Tepoztlán & $\mathrm{AB}$ & $\mathrm{MC}$ & $17.5 \pm 0.6^{\mathrm{c}}$ & $\mathrm{Ar}-\mathrm{Ar}$ & 10 \\
\hline 1920 & SLT & $99^{\circ} 05^{\prime} 5.25^{\prime \prime}$ & $19^{\circ} 22^{\prime} 44.27^{\prime \prime}$ & Formación Tepoztlán & $\mathrm{AB}$ & $\mathrm{MC}$ & $18.3 \pm 0.4^{\mathrm{c}}$ & $\mathrm{Ar}-\mathrm{Ar}$ & 10 \\
\hline 2002 & SLT & $99^{\circ} 05^{\prime} 5.25^{\prime \prime}$ & $19^{\circ} 22^{\prime} 44.27^{\prime \prime}$ & Formación Tepoztlán & A & $\mathrm{MC}$ & $20.1 \pm 0.3^{\mathrm{c}}$ & $\mathrm{Ar}-\mathrm{Ar}$ & 10 \\
\hline 837 & Texcoco-1 & $98^{\circ} 59.9^{\prime} \S$ & $19^{\circ} 27.3^{\prime} \S$ & & A \# & $\mathrm{RT}$ & $15.8 \pm 2.35 \diamond$ & $\mathrm{K}-\mathrm{Ar}$ & 1 \\
\hline $950 \bullet$ & Texcoco-1 & $98^{\circ} 59.9^{\prime} \S$ & $19^{\circ} 27.3^{\prime} \S$ & & A \# & RT & $24.05 \pm 1.6 \diamond$ & $\mathrm{K}-\mathrm{Ar}$ & 1 \\
\hline 1520 & Texcoco-1 & $98^{\circ} 59.9^{\prime} \S$ & $19^{\circ} 27.3^{\prime} \S$ & & B-A \# & RT & $30.8 \pm 0.35 \diamond$ & $\mathrm{K}-\mathrm{Ar}$ & 1 \\
\hline $525-532$ & Copilco-1 & $99^{\circ} 10^{\prime} 22.09^{\prime \prime}$ & $19^{\circ} 18^{\prime} 50.09^{\prime \prime}$ & & B & RT & $0.761 \pm 0.06$ & $\mathrm{~K}-\mathrm{Ar}$ & $2,3 a, 4,11$ \\
\hline $1700-1709$ & Copilco-1 & $99^{\circ} 10^{\prime} 22.09^{\prime \prime}$ & $19^{\circ} 18^{\prime} 50.09^{\prime \prime}$ & & B & $\mathrm{Plg}$ & $12.3 \pm 1.3$ & $\mathrm{~K}-\mathrm{Ar}$ & $2,3 \mathrm{a}, 4,11$ \\
\hline $2150-2157$ & Copilco-1 & $99^{\circ} 10^{\prime} 22.09^{\prime \prime}$ & $19^{\circ} 18^{\prime} 50.09^{\prime \prime}$ & & $\mathrm{CA}$ & RT & $13.7 \pm 0.8$ & $\mathrm{~K}-\mathrm{Ar}$ & $2,3 \mathrm{a}, 4,11$ \\
\hline $2250-2258$ & Copilco-1 & $99^{\circ} 10^{\prime} 22.09^{\prime \prime}$ & $19^{\circ} 18^{\prime} 50.09^{\prime \prime}$ & & B & RT & $23.3 \pm 1.5$ & $\mathrm{~K}-\mathrm{Ar}$ & $2,3 \mathrm{a}, 4,11$ \\
\hline $1263-1272$ & Roma-1 & $99^{\circ} 09^{\prime} 26.08^{\prime \prime}$ & $19^{\circ} 24^{\prime} 33.90^{\prime \prime}$ & & B & RT & $8.7 \pm 0.7$ & $\mathrm{~K}-\mathrm{Ar}$ & $3 b, 4,11$ \\
\hline $2207-2210$ & Roma-1 & $99^{\circ} 09^{\prime} 26.08^{\prime \prime}$ & $19^{\circ} 24^{\prime} 33.90^{\prime \prime}$ & & B & RT & $25.9 \pm 2.0$ & $\mathrm{~K}-\mathrm{Ar}$ & $3 b, 4,11$ \\
\hline $100-109$ & Mixhuca-1 & $99^{\circ} 05^{\prime} 13.57^{\prime \prime}+$ & $19^{\circ} 23^{\prime} 41.52^{\prime \prime}+$ & & B & RT & $1.2 \pm 0.1$ & $\mathrm{~K}-\mathrm{Ar}$ & 2,4 \\
\hline $1225-1231$ & Mixhuca-1 & $99^{\circ} 05^{\prime} 13.57^{\prime \prime}$ * & $19^{\circ} 23^{\prime} 41.52^{\prime \prime} \ddagger$ & & B & RT & $21.7 \pm 1.3$ & $\mathrm{~K}-\mathrm{Ar}$ & 2,4 \\
\hline $1281-1281.4$ & Mixhuca-1 & $99^{\circ} 05^{\prime} 13.57^{\prime \prime}+$ & $19^{\circ} 23^{\prime} 41.52^{\prime \prime}+$ & & A & RT & $29 \pm 2.0$ & $\mathrm{~K}-\mathrm{Ar}$ & 2,4 \\
\hline $1122-1131$ & Tulyehualco-1 & $99^{\circ} 03^{\prime} 37.46^{\prime \prime}$ & $19^{\circ} 16^{\prime} 46.95^{\prime \prime}$ & & A & RT & $15.0 \pm 0.7$ & $\mathrm{~K}-\mathrm{Ar}$ & $2,3 \mathrm{c}, 4,11$ \\
\hline $1327-1336$ & Tulyehualco-1 & $99^{\circ} 03^{\prime} 37.46^{\prime \prime}$ & $19^{\circ} 16^{\prime} 46.95^{\prime \prime}$ & & B & RT & $12.2 \pm 0.6$ & $\mathrm{~K}-\mathrm{Ar}$ & $2,3 \mathrm{c}, 4,11$ \\
\hline $1897-1899$ & Tulyehualco-1 & $99^{\circ} 03^{\prime} 37.46^{\prime \prime}$ & $19^{\circ} 16^{\prime} 46.95^{\prime \prime}$ & & A & RT & $11.8 \pm 1.9$ & $\mathrm{~K}-\mathrm{Ar}$ & $2,3 \mathrm{c}, 4,11$ \\
\hline
\end{tabular}

$\mathrm{CVH}=$ Campo Volcánico de Huautla; SLT $=$ San Lorenzo Tezonco.

$\mathrm{B}=$ basalto; $\mathrm{AB}=$ andesita basáltica; $\mathrm{A}=$ andesita; $\mathrm{CA}=$ clasto de andesita en una roca piroclástica o toba; $\mathrm{D}=$ dacita; $\mathrm{Di}=$ diorita: $\mathrm{IR}=$ ignimbrita riolítica; $\mathrm{RT}=$ roca total; $\mathrm{MC}=$ muestra de canal; $\mathrm{zr}=$ zircón; $\mathrm{plg}=$ plagioclasa; $\mathrm{FT}=$ Formación Tepoztlán.

$\mathrm{c}=$ edad de isocrona; Arce et al. (2013a) considera que las edades Ar-Ar en letras negritas tienen mayor exactitud.

- Marsal y Graue (1969)

§ Coordenadas estimadas de la figura 2 de Vázquez-Sánchez y Jaimes-Palomera (1989) y la figura 6 de Pérez-Cruz (1988)

\# Oviedo de León (1967)

$\checkmark$ errores inferidos en el presente trabajo, considerando las edades publicados en SHCP (1969) y los rangos de edades publicados en Marsal y Graue (1969) $\$$ CONAGUA, SEMARNAT (2013)

Referencias: 1. Secretaría de Hacienda y Crédito Público (1969); 2. Murillo-M. y Torres-V. (1987); 3. PEMEX (1987a, b, c, en Pérez-Cruz, 1988 ); 4. Pérez-Cruz (1988); 5. García Palomo (1998); 6. García-Palomo et al. (2000); 7. Lenhardt et al. (2010); 8. Arce et al. (2013a); 9. González-Torres et al. (2013); 10. Arce et al., 2015; 11. G. Pérez-Cruz, comunicación personal, abril de 2015; 12. Lenhart, comunicación personal, mayo de 2015. 


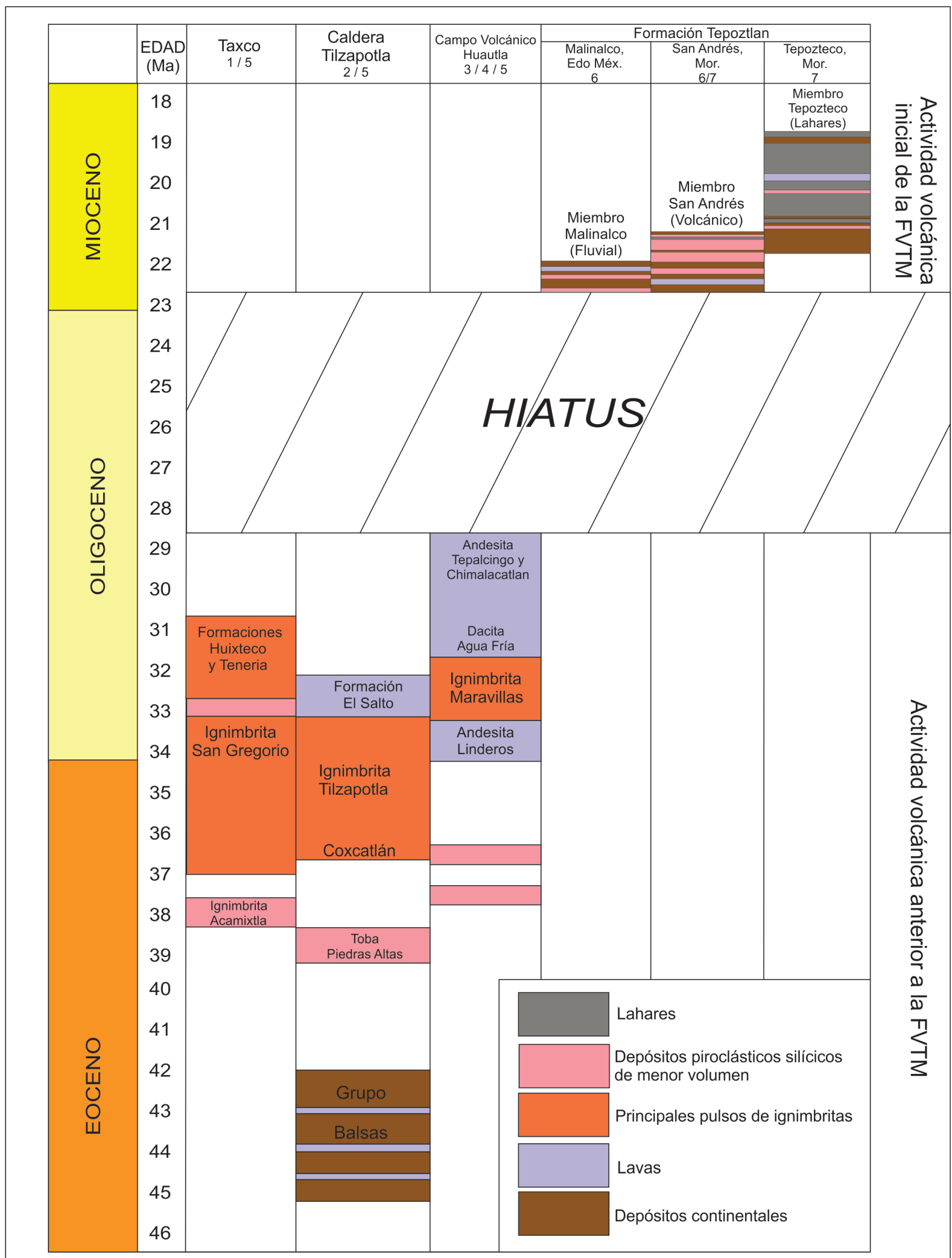

1/ Alaniz-Álvarez et al., 2002; 2/ Morán-Zenteno et al., 2004, 2007; 3/ Cerca et al., 2007; 4/ Campa-Uranga et al., 2002; 5/ González-Torres et al., 2013; 6/ García-Palomo et al., 2000; 7/ Lenhardt et al., 2010.

Figura 5. Síntesis del registro estratigráfico de la región ubicada al sur de la Cuenca de México, en donde se muestran los principales eventos de actividad volcánica anterior a la FVTM de los campos volcánicos de Huautla, Morelos y Taxco, Guerrero, de la caldera de Tilzapotla, Guerrero, así como de la actividad volcánica inicial de la FVTM en el sector de Malinalco, Estado de México y de la región de Tepoztlán, Morelos. Observar que el hiatus entre la declinación de la actividad volcánica de la Sierra Madre del Sur y de los primeros episodios de la formación de la FVTM es menor que el rango de edad considerado anteriormente. 
Tepoztlán correspondiente a los primeros episodios de la FVTM, se observa un hiatus de la actividad volcánica de casi $6 \mathrm{Ma}$, ubicado entre los $\sim 28.5$ y $22.8 \mathrm{Ma}$.

\section{Discusión y conclusiones}

Para establecer las relaciones entre el subsuelo de la CM y el registro volcánico cenozoico ubicado en el norte de la SMS, se comparan las características litológicas y la cronología de las sucesiones volcánicas cenozoicas de ambas regiones.

La perforación del pozo Texcoco-1 permitió conocer las características del subsuelo profundo de un sector de la $\mathrm{CM}$, haciendo necesario revisar las consideraciones que se tenían sobre su edad y origen (Oviedo de León, 1970). En particular, la edad K-Ar de 30.8 Ma obtenida en un cuerpo volcánico de la parte inferior de la columna (SHCP, 1969) permitió reconocer que el relleno volcánico de la CM tenía un registro estratigráfico por lo menos a partir del Oligoceno inferior.

Asimismo, el pozo Roma-1, que perforó 3200 m sin cortar el basamento Mesozoico, permitió reconocer que el basamento mesozoico de la CM podría alcanzar los 4 km de profundidad (Pérez-Cruz, 1988; Alaniz-Álvarez y Nieto-Samaniego, 2005).

La información reciente sobre la litología y las edades obtenidas en el pozo San Lorenzo Tezonco, así como el mayor detalle cronológico de las unidades que constituyen el entorno geológico (Arce et al., 2013a, 2015 y referencias citadas, Lenhardt et al., 2010; Morán-Zenteno et al., 2007; González-Torres et al., 2013), indican que la agrupación de las unidades volcánicas propuesta por Pérez-Cruz (1988) no es del todo funcional para realizar correlaciones de mayor detalle con unidades estratigráficas del entorno de la $\mathrm{CM}$ (Figura 5).

En este trabajo se reconoce que el relleno de la $\mathrm{CM}$ se puede agrupan en varios eventos volcánicos. 1) El evento del Oligoceno es el más antiguo; está definido por las edades K-Ar de 30.8 y 29 Ma obtenidas en unas lavas andesíticas y basálticas cortadas por el pozo Texcoco-1 a una profundidad un poco mayor a $1500 \mathrm{~m}$, y por el pozo Mixhuca-1 a $1281 \mathrm{~m}$ de profundidad ( $200 \mathrm{~m}$ arriba de las secuencias de calizas cretácicas); este evento se puede correlacionar con el magmatismo del campo volcánico de Huautla (González-Torres et al., 2013). 2) El evento del Mioceno inferior está representado por lavas andesíticas $\mathrm{y}$ andesitas basálticas con edades variables entre 24.05 y 18.4 Ma, reportadas en los pozos Copilco-1, Mixhuca-1, San Lorenzo Tezonco y Texcoco-1 (Figura 3, Tabla 1). Este evento se correlaciona cronológicamente con los primeros episodios magmáticos de la FVTM, representados por la Formación Tepoztlán y la andesita-basáltica San Nicolás (García-Palomo et al., 2000; Ferrari et al., 2002; Lenhardt et al., 2010). Cabe señalar que Arce et al. (2015) extienden la correlación de la Formación Tepoztlán con rocas más jóvenes de hasta $\sim 13$ Ma reconocidas en el subsuelo de la CM. En virtud de que esta correlación se extiende a edades más jóvenes que las reportadas en la Formación Tepoztlán (Lenhardt et al., 2010), en este trabajo se prefiere utilizar la agrupación que se menciona arriba. 3) El evento del Mioceno medio-inicio del Mioceno tardío está constituido por lavas andesíticas y dacíticas con edades variables de $\sim 17$ a $8.7 \mathrm{Ma}$; estos productos se correlacionan con las Andesitas Apan y el Peñón que afloran en el norte de la CM (García-Palomo et al., 2002). 4) Los eventos del PlioCuaternario están constituidos por lavas de composición variable desde andesita-basáltica a dacita, ignimbritas, conglomerados y brechas volcánicas procedentes de las Sierras Chichinautzin, las Cruces y Nevada (Arce et al., 2013b, 2015, García-Palomo et al., 2008; Macías et al., 2012 y referencias citadas); estos productos, con edades variables desde 5 a $0.5 \mathrm{Ma}$, sólo se han reportado en los pozos Copilco-1 y San Lorenzo Tezonco (Murillo-M. y Torres-V., 1987; Arce et al., 2013a) (Figura 3).

La formación de una cuenca continental volcanosedimentaria con las características de la $\mathrm{CM}$, requiere la intervención de dos factores: por una parte, un proceso de subsidencia continúa asociado a una tectónica de extensión; por otra, el aporte de materiales para su relleno. La información sobre la litología y geocronología de los materiales que constituyen el relleno de la CM descrito anteriormente (Pérez-Cruz, 1988; Arce et al., 2013a) indica que el mayor espesor de materiales acumulados corresponde a sucesiones del Mioceno al Cuaternario. La ausencia de espesores significativos de rocas volcánicas o sedimentarias con edades probables del Paleógeno entre las sucesiones del Neógeno y las capas marinas del Cretácico en el subsuelo de la CM sugiere que la subsidencia ocurrió principalmente durante el Neógeno. Esta interpretación se ve reforzada por el hecho de que durante los últimos episodios de sedimentación y volcanismo del Oligoceno temprano de la parte norte de la SMS no se registran episodios de extensión con subsidencia; en contraste, el régimen tectónico dominante produjo fallas de desplazamiento lateral izquierdo con una orientación preferencial NW-SE (Alaniz-Álvarez et al., 2002; MoránZenteno et al., 2004, 2007; González-Torres et al., 2013) (Figura 4). En la porción norte-central de la SMS se han documentado acumulaciones sedimentarias continentales y volcánicas del Grupo Balsas del Paleógeno en cuencas como las de Amacuzac, Tuzantlán y Copalillo, que son de tipo intermontano, formadas a partir del relieve irregular heredado de las estructuras erosionadas de pliegues y cabalgaduras del Cretácico Tardío. En el caso de la región de Taxco, la subsidencia se vio favorecida por la extensión tectónica en una zona de transferencia de sistemas de fallas laterales (Alaniz-Álvarez et al., 2002). Dentro del contexto anterior, es posible que los primeros eventos de acumulación sedimentaria y volcánica del subsuelo de la CM todavía estuvieran asociados a la deformación transcurrente que se ha documentado para el sector norte-central de la SMS durante el Eoceno e inicios del Oligoceno. 
Se ha argumentado que la formación de los campos volcánicos de Taxco, Guerrero y Huautla, así como la caldera de Tilzapotla, se llevó a cabo en un intervalo de tiempo entre los 38 y $28 \mathrm{Ma}$, lapso durante el cual se registran varios episodios de actividad volcánica explosiva que constituyen un "flare up" en el sector norte-central de la SMS (González-Torres et al., 2013). Asimismo, la declinación de la actividad volcánica de la SMS en este sector está representada por derrames de rocas volcánicas con rangos de edad que van de los $\sim 32$ a los $28 \mathrm{Ma}$ (MoránZenteno et al., 2004; González-Torres et al., 2013).

En forma particular, el campo volcánico de Huautla, en donde se encuentran expuestos los últimos episodios de actividad volcánica de la región, es él que, por sus características litológicas y edad, tiene correlación con las unidades volcánicas más profundas del pozo Texcoco-1 (de donde se obtuvo una edad K/Ar de $30.8 \mathrm{Ma}$ ) y Mixhuca-1 (donde se reporta una edad de $29 \pm 2 \mathrm{Ma}$ ) (SHCP, 1969; Pérez-Cruz, 1988). Estas posibles interpretaciones están sujetas a una revisión y a nuevos fechamientos basados en concentrados minerales y métodos más modernos como Ar-Ar y U-Pb LA-ICPMS.

En el pozo Roma-1 se registra un espesor de un poco más de $800 \mathrm{~m}$, debajo de la unidad más antigua que se fechó, de la cual se obtuvo una edad por K-Ar de $25.9 \pm 2.0 \mathrm{Ma}$. Con base en esto, es posible que las unidades volcánicas más profundas puedan alcanzar edades semejantes a las reportadas en los campos volcánicos de Huautla, Taxco y en la caldera de Tilzapotla.

En lo referente a la actividad más antigua de la FVTM en los sectores de Malinalco y Tepoztlán, se han reportado rocas volcánicas con un rango de edad entre $21.6 \pm 1 \mathrm{y}$ 18.8 Ma: la litología y cronología de estas sucesiones muestran estrechas similitudes con lo reportado en los pozos Texcoco-1, Copilco-1, Mixhuca-1 y San Lorenzo Tezonco (Pérez-Cruz, 1988; Arce et al., 2013a, 2015); dichas afinidades temporales han sido también reconocidas por Arce et al. (2015).

Un aspecto sobresaliente en términos de los contrastes tectónicos entre la CM y el norte de la SMS es el considerable desnivel entre las capas de calizas marinas y yesos del Cretácico que constituyen el basamento de la CM, y la altitud de las capas de calizas marinas ubicadas entre Cuernavaca y Yautepec. Las primeras se encuentran a una altitud entre $\sim 100$ y $600 \mathrm{~m}$ sobre el nivel del mar; mientras que los afloramientos de las segundas se encuentran a una altitud cercana a los $1800 \mathrm{~m}, \mathrm{y}$ a una distancia de $\sim 40 \mathrm{~km}$ al sur de la CM. Lo anterior indica que el bloque ubicado al norte del sistema E-W del horst de Chichinautzin (Figura 2) representa un bloque caído con una subsidencia rellenada por al menos de $1600 \mathrm{~m}$ de sucesiones volcánicas y depósitos lacustres (Pérez-Cruz, 1988, Ferrari et al., 2003; Siebe et al., 2004a; Alaniz-Álvarez y Nieto-Samaniego, 2005; Lozano-García y Ortega-Guerrero, 1998).

El horst de Chichinautzin representa una frontera tectónica para el Mioceno, ya que mientras al norte de esta estructura, en el subsuelo de la CM, se puede documentar una subsidencia pronunciada, al sur no hay evidencia de estructuras tectónicas de extensión y subsidencia notables (Morán-Zenteno et al., 2005). Al menos desde el Oligoceno temprano $(<28 \mathrm{Ma})$, el régimen tectónico en el norte de la SMS se ha caracterizado por la exhumación, sin desarrollo de cuencas de extensión con subsidencia. Aunque no hay datos que permitan confirmar un levantamiento real de esta región, la exhumación se puede documentar por la profunda disección erosiva del norte de la SMS debida a la erosión en las cabeceras de los afluentes del río Balsas. Esta disección sólo fue temporalmente interrumpida durante el Mioceno y Plioceno por la acumulación de flujos de escombros procedentes de la FVTM (Formaciones Tepoztlán y Cuernavaca) (Arce et al., 2013b, Lenhardt et al., 2010).

El hiatus magmático entre el volcanismo paleógeno de la SMS y la sucesión miocénica de Tepoztlán $(28-23 \mathrm{Ma})$ parece responder a un cambio importante en la geometría de la placa subducida en el sur de México. A partir del análisis de la configuración actual de la zona de subducción del sur de México, se reconoce un segmento de placa subhorizontal en la base de la corteza continental desde la región de la costa hasta el norte de Iguala (Pérez-Campos et al., 2008). Es evidente que la evolución de la FVTM se inició a partir de un cambio radical de la geometría de la placa, con respecto a la que prevaleció hasta el Oligoceno temprano (Morán-Zenteno et al., 2000, Ferrari et al., 2014. El registro abundante del magmatismo Paleógeno de arco desde la margen continental truncada actual hasta latitudes cercanas a Cuernavaca indica que en el lugar del segmento plano de la zona de subducción actual, se encontró una cuña del manto productiva que dio origen al magmatismo de la SMS (Mori et al., 2012). El escenario descrito permite entender las diferencias geológicas, tectónicas y temporales que existen entre la FVTM de la SMS. Existen dos fechas $\mathrm{K}-\mathrm{Ar}$ en roca total con errores relativamente grandes de rocas volcánicas de los pozos Texcoco-1 y Roma-1 (24.1 y $25.9 \mathrm{Ma}$, Tabla 1) que parecen romper con este hiatus, por lo cual se requiere una revisión de la geocronología de estos materiales o un replanteamiento de la hipótesis. Aunque la información aún es insuficiente, los datos disponibles sobre la composición, la geocronología y la deformación que acompaño al magmatismo de la SMS y de la CM indican una transición entre las dos provincias de las condiciones tectónico-magmáticas del sur de México debido a cambios profundos en la geometría de la subducción.

\section{Agradecimientos}

Los autores agradecen el apoyo en la elaboración de algunas figuras a Carolina Castelán, Sergio Macías Medrano, y Juan Carlos Alvarado Sánchez. Asimismo, agradecemos al Dr. Antonio Hernández-Espriú las facilidades para consultar las imágenes de varios modelos de elevación digital, así como los comentarios de Rodrigo Gutiérrez, Aline Concha 
y Alberto Arias y Javier Mancera. La revisión realizada por el Dr. Luis Delgado y dos árbitros anónimos, contribuyeron sustantivamente a mejorar el manuscrito.

\section{Referencias}

Aguayo-Camargo, J.E., Marín-Córdova, S., Sánchez-Díaz, F., 1989, Evolución geológica de la Cuenca de México: Boletín de la Sociedad Mexicana de Mecánica de Suelos. Tópicos geológicos de la Cuenca del Valle de México: Memoria de Simposio, 25-41.

Alaniz-Álvarez, S.A., Nieto-Samaniego, A.F., Morán-Zenteno, D.J., AlbaAldave, L., 2002, Rhyolitic volcanism in extension zone associated with strike-slip tectonics in the Taxco region, southern Mexico: Journal of Volcanology and Geothermal Research, 118, 1-14.

Alaniz-Álvarez, S.A., Nieto-Samaniego, A.F., 2005, El sistema de fallas Taxco-San Miguel de Allende y la Faja Volcánica Transmexicana, dos fronteras tectónicas del centro de México activas durante el Cenozoico: Boletín de la Sociedad Geológica Mexicana, 57 (1), 65-82.

Arce, J.L., Macías, R., García-Palomo, A., Capra, L., Macías, J.L., Layer, P., Rueda, H., 2008, Late Pleistocene flank collapse of Zempoala volcano (Central Mexico) and the role of fault reactivation: Journal of Volcanology and Geothermal Research, 177, 944-958.

Arce, J.L., Layer, P.W., Morales-Casique, E., Benowitz, J.A., Rangel, E., Escolero, O., 2013a, New constraints on the subsurface geology of the Mexico City Basin: The San Lorenzo Tezonco deep well, on the basis of ${ }^{40} \mathrm{Ar} /{ }^{39} \mathrm{Ar}$ geochronology and whole-rock chemistry: Journal of Volcanology and Geothermal Research, 266, 34-49.

Arce, J.L., Layer, P.W., Lassiter, J.C., Benowitz, J.A., Macias, J.L., Ramírez-Espinosa, J., 2013b, ${ }^{40} \mathrm{Ar} /{ }^{39} \mathrm{Ar}$ dating, geochemistry, and isotopic analyses of the quaternary Chichinautzin volcanic field, south of Mexico City: implications for timing, eruption rate, and distribution of volcanism: Bulletin of Volcanology, 75, 774.

Arce, J.L., Layer, P., Martínez, I., Salinas, J.I., Macías-Romo, M.C., Morales-Cacique, E., Benowitz, J., Escolero, O., Lenhardt, N., 2015, Geología y estratigrafía del pozo profundo San Lorenzo Tezonco y sus alrededores, sur de la Cuenca de México: Boletín de la Sociedad Geológica Mexicana, 67 (2), 123-143.

Cabral-Cano, E., Dixon, T.H., Miralles-Wilhelm, F., Díaz-Molina, O., Sánchez-Zamora, O., Carande, R.E., 2008, Space geodetic imaging of rapid ground subsidence in Mexico City: Geological Society of America Bulletin, 120 (11/12), 1556-1566.

Campa-Uranga, M.F., Fitz-Díaz, E., Martínez-Hernández, E., 2002, Revisión de la edad de la Formación Oapan y su significado en el graben de San Agustín Oapan y el sinclinorio de Zacango, Estado de Guerrero (resumen), en Unión Geofísica Mexicana, III Reunión Nacional de Ciencias de la Tierra: GEOS, 22 (2), 165.

Campos-Enríquez, J.O., Alatriste-Vilchis, D.R., Huizar-Álvarez, R., Marines-Campos, R., Alatorre-Zamora, M.A., 2003, Subsurface structure of the Tecocomulco sub-basin (northeastern Mexico basin), and its relationship to regional tectonics: Geofísica Internacional, 42 (1), 3-24.

Cerca, M., Ferrari, L., López-Martínez, M., Martiny, B., Iriondo, A., 2007, Late Cretaceous shortening and early Tertiary shearing in the central Sierra Madre del Sur, southern Mexico: Insights into the evolution of the Caribbean-North America plate interaction: Tectonics, 26, TC3007, doi:10.1029/2006TC001981.

Chávez-Pérez, S., 1993, Modelado sísmico de la cuenca de la ciudad de México: Geofísica Internacional, 32 (1), 111-130.

Colín-Rodríguez, A., 2006, Neotectónica de la Semifosa TláhuacTulyehualco: México D.F., Instituto Politécnico Nacional, Escuela Superior de Ingeniería y Arquitectura, tesis de licenciatura, 75 p.

CONAGUA, SEMARNAT, 2013, Avance del estudio de los acuíferos profundos del Valle de México, México D.F., 31 de octubre de 2013, disponible en <http://www.conagua.gob.mx/conagua07/ notap/Exploracion \%20Aguas \%20Profundas\%20V\%20de $\% 20$ M\%20\%20Evento\%20CONAGUA-SACM-1.pdf>, consultado 6 de abril de 2015 .

de Cserna, Z., de la Fuente-Duch, M., Palacios-Nieto, M., Triay, L., MitreSalazar, L.M., Mota-Palomino, R., 1988, Estructura geológica, gravimetría, sismicidad y relaciones neotectónicas regionales de la Cuenca de México: México, D.F., Universidad Nacional Autónoma de México, Instituto de Geología, Boletín 104, 71 p.

Delgado-Granados, H., Nieto-Obregón, J., Silva-Romo, G., MendozaRosales, C., Arellano-Gil, J., Lermo-Samaniego, J.F., RodríguezGonzález., M., 1995, La Pera detachment fault system: active faulting south of Mexico City: geological evidence (II) (resumen), en Reunión Anual de la Unión Geofísica Mexicana, Puerto Vallarta, Jalisco, GEOS, 15 (2), 64.

Enciso-De la Vega, S., 1992, Propuesta de nomenclatura estratigráfica para la cuenca de México: México, Universidad Nacional Autónoma de México, Instituto de Geología, Revista, 10 (1), 26-36.

Ferrari, L., Mena, M., López-Martínez, M., Jacobo-Albarrán, J., SilvaRomo, G., Mendoza-Rosales, C., González-Cervantes, N., 2002, Estratigrafía y tectónica de la cuenca de la Ciudad de México y áreas colindantes (resumen), en Tercera Reunión Nacional de Ciencias de la Tierra, Puerto Vallarta, Jalisco, GEOS, 22 (2), 150.

Ferrari, L., López-Martínez, M., González-Cervantes, N., Jacobo-Albarrán, J., Hernández-Bernal, M.S., 2003, Volcanic record and age of formation of the Mexico City Basin (resumen), en Reunión Anual de la Unión Geofísica Mexicana, GEOS, 23 (2), 120.

Ferrari, L., Orozco-Esquivel, T., Manea, V., Manea, M., 2012, The dynamic history of the Trans-Mexican Volcanic Belt and the Mexico subduction zone: Tectonophysics, 522-523, 122-149.

Ferrari, L., Bergomi, M., Martini, M., Tunesi, A., Orozco-Esquivel, T., López-Martínez, M., 2014, Late Cretaceous-Oligocene magmatic record in southern Mexico: The case for a temporal slab window along the evolving Caribbean-North America-Farallon triple boundary: Tectonics, 33, 1738-1765, doi: 10.1002/2014TC003525.

Fries, C., Jr., 1960, Geología del Estado de Morelos y de partes adyacentes de México y Guerrero, región central-meridional de México: Universidad Nacional Autónoma de México, Instituto de Geología, Boletín 60, 236 p.

García-Palomo, A., 1998, Evolución estructural en las inmediaciones del Volcán Nevado de Toluca, Estado de México: México, D.F., México, UACPyP del CCH, Universidad Nacional Autónoma de México, Tesis de Maestría, 146 p.

García-Palomo, A., Macías, J.L., Garduño, V.H., 2000, Miocene to Recent structural evolution of the Nevado de Toluca volcano region, Central Mexico: Tectonophysics, 318, 281-302.

García-Palomo, A., Macías, J.L., Tolson, G., Valdez, G., Mora, J.C., 2002, Volcanic, stratigraphy and geological evolution of the Apan region, east-central sector of the Trans-Mexican Volcanic Belt: Geofísica Internacional, 41 (2), 133-150.

García-Palomo, A., Zamorano, J.J., López-Miguel, C., Galván-García, A., Carlos-Valerio, V., Ortega, R., Macías, J.L., 2008, El arreglo morfoestructural de la Sierra de Las Cruces, México central: Revista Mexicana de Ciencias Geológicas, 25 (1), 158-178.

Gómez-Tuena, A., Orozco-Esquivel, M.T., Ferrari, L., 2005, Petrogénesis ígnea de la Faja Volcánica Transmexicana: Boletín de la Sociedad Geológica Mexicana, Volumen Conmemorativo del Centenario, Temas Selectos de la Geología Mexicana, 57 (3), 227-285.

González-Torres, E.A., Morán-Zenteno, D.J., Mori, L., Díaz-Bravo, B., Martiny, B.M., Solé, J., 2013, Geochronology and magmatic evolution of the Huautla volcanic field: last stages of extinct Sierra Madre del Sur igneous province of southern Mexico: International Geology Review, 55 (9), 1145-1161.

Hiriart, F., Graue, R., 1969, Proyecto Texcoco, en El hundimiento de la Ciudad de México y Proyecto Texcoco, Volumen Nabor Carrillo: México, D.F., Secretaría de Hacienda y Crédito Público, 149-165.

Instituto Nacional de Estadística y Geografía (INEGI), 2013, Perfil Sociodemográfico, Estados Unidos Mexicanos, Censo de Población y Vivienda 2010 (en línea), disponible en $<\mathrm{http}$ :/www. inegi.org.mx/prod_serv/contenidos/espanol/bvinegi/productos/ censos/poblacion/2010/perfil_socio/uem/702825047610_1.pdf>, consultado 7 de septiembre de 2014 . 
Lenhardt, N., Böhnel, H., Wemmer, K., Torres-Alvarado, I.S., Hornung, J., Hinderer, M., 2010, Petrology, magnetostratigraphy and geochronology of the Miocene volcaniclastic Tepoztlán Formation: implications for the initiation of the Transmexican Volcanic Belt (Central Mexico): Bulletin of Volcanology, 72, 817-832.

Lozano-García, M.S., Ortega-Guerrero, B., 1998, Late Quaternary environmental changes of the central part of the Basin of Mexico; correlation between Texcoco and Chalco basins: Review of Palaeobotany and Palynology, 99, 77-93.

Lugo-Hubp, J., Mooser, F., Pérez-Vega, A., Zamorano-Orozco, J., 1994, Geomorfología de la Sierra de Santa Catarina, D.F., México: Revista Mexicana de Ciencias Geológicas, 11 (1), 43-52.

Macías, J.L., Arce, J.L., García-Tenorio, F., Layer, P.W., Rueda, H., Reyes-Agustín, G., López-Pizaña, F., Avellán, D., 2012, Geology and geochronology of Tlaloc, Telapón, Iztaccíhuatl and Popocatépetl volcanoes, Sierra Nevada, central Mexico, en Aranda-Gómez, J.J., Tolson, G., Molina-Garza, R.S., (eds.), The southern Cordillera and Beyond: Geological Society of America, Field Guide 25, 163-193.

Marsal, R.J., Graue, R., 1969, El subsuelo del lago de Texcoco, en Carrillo Nabor, El hundimiento de la Ciudad de México y Proyecto Texcoco, México, D.F., Secretaria de Hacienda y Crédito Público, 167-202.

Mooser, F., 1975, Historia Geológica de la Cuenca de México, in Memoria de las obras del sistema de drenaje profundo del Distrito Federal: México, D.F., Departamento del Distrito Federal, 1, 7-38.

Mooser, F., Montiel, A., Zuñiga, A., 1996, Nuevo mapa geológico de las cuencas de México, Toluca y Puebla. Estratigrafía, tectónica regional y aspectos geotérmicos: México, Comisión Federal de Electricidad, 36 mapas, Texto explicativo $36 \mathrm{p}$.

Morán-Zenteno, D.J., Martiny, B., Tolson, G., Solís-Pichardo, G., AlbaAldave, L., Hernández-Bernal, M.S., Macías-Romo, C., MartínezSerrano, R.G., Schaaf, P., Silva Romo, G., 2000, Geocronología y características geoquímicas de las rocas magmáticas terciarias de la Sierra Madre del Sur: Boletín de la Sociedad Geológica Mexicana, $53(1), 27-58$.

Morán-Zenteno, D.J., Alba-Aldave, L.A., Solé, J., Iriondo, A., 2004, A major resurgent caldera in southern Mexico: the source of late Eocene Tilzapotla ignimbrite: Journal of Volcanology and Geothermal Research, 136, 97-119.

Morán-Zenteno, D.J., Cerca, M., Keppie, J.D., 2005, La evolución tectónica y magmática cenozoica del suroeste de México: avances y problemas de interpretación, Boletín de la Sociedad Geológica Mexicana, 57 (3), 319-341.

Morán-Zenteno, D.J., Monter-Ramírez, A., Centeno-García, E., AlbaAldave, L.A., Solé, J., 2007, Stratigraphy of the Balsas Group in the Amacuzac area, southern Mexico: relationship with Eocene volcanism and deformation of the Tilzapotla-Taxco sector: Revista Mexicana de Ciencias Geológicas, 24 (1), 68-80.

Mori, L., Morán-Zenteno, D.J., Martiny, B.M., González-Torres, E.A., Chapela-Lara, M., Díaz-Bravo, B., Roberge, J., 2012, Thermomechanical maturation of the continental crust and its effects on the late Eocene-early Oligocene volcanic record of the Sierra Madre del Sur Province, southern Mexico: International Geology Review, 54 (13), 1475-1496.

Murillo-M., G., Torres-V., R., 1987, Mapa petrogenético y radiométrico de la República Mexicana, Proyecto C-2010: Instituto Mexicano del Petróleo, Subdirección de Tecnología de Exploración, 78 p., con Apéndices.
Norini, G., Groppelli, G., Lagmay, A.M.F., Capra, L., 2006, Recent leftoblique slip faulting in the central eastern Trans-Mexican Volcanic Belt: Seismic hazard and geodynamic implications: Tectonics, 25 (4). doi: 10.1029/2005TC001877.

Oviedo de León, A., 1967, Estudio geológico del subsuelo, basado en los datos obtenidos de la perforación del pozo profundo Texcoco No. 1: Instituto Mexicano de Petróleo, Departamento de Geología y Exploración, 1-18, con Apéndices.

Oviedo de León, A., 1970, El Conglomerado Texcoco y el posible origen de la Cuenca de México: Revista del Instituto Mexicano del Petróleo, 2, 5-20.

Pérez-Cruz, G.A., 1988, Estudio sismológico de reflexión del subsuelo de la Ciudad de México: México, D.F., Universidad Nacional Autónoma de México, Tesis de Maestría, División de Estudios de Posgrado de la Facultad de Ingeniería, $83 \mathrm{p}$.

Pérez-Campos, X., Hee Kim, Y., Husker, A., Davis, P., Clayton, R., Iglesias, A., Pacheco, J., Singh, S., Manea, V., Gurnis, M., 2008, Horizontal subduction and truncation of the Cocos Plate beneath central Mexico: Geophysical Research Letters, 35, L18305

Petróleos Mexicanos (PEMEX), 1987a, Informe geológico final del pozo Copilco-1. Inédito.

Petróleos Mexicanos (PEMEX), 1987b, Informe geológico final del pozo Roma-1. Inédito.

Petróleos Mexicanos (PEMEX), 1987c, Informe geológico final del pozo Tulyehualco-1. Inédito.

Santoyo-Villa, E., Ovando-Shelley, E., Mooser, F., León-Plata, E., 2005, Síntesis geotécnica de la cuenca del Valle de México: México, D.F., TGC Geotécnia, $171 \mathrm{p}$.

Schlaepfer, C.J., 1968, Hoja México 14Q-h(5) con Resumen de la Geología de la Hoja México, Distrito Federal y Estados de México y Morelos: México, D.F., Universidad Nacional Autónoma de México, Instituto de Geología, Carta Geológica de México serie de 1:100000, 1 mapa con texto.

Secretaría de Hacienda y Crédito Público (SHCP), 1969, Proyecto Texcoco: Memoria de los trabajos realizados y conclusiones: México, D.F., Nacional Financiera, S.A., 215 p.

Siebe, C., Rodríguez-Lara, V., Schaaf, P., Abrams, M., 2004a, Radiocarbon ages of Holocene Pelado, Guespalapa, and Chichinautzin scoria cones, south of Mexico City: implications for archaeology and future hazards: Bulletin of Volcanology, 66, 203-225.

Siebe, C., Rodríguez-Lara, V., Schaaf, P., Abrams, M., 2004b, Geochemistry, Sr-Nd isotope composition, and tectonic setting of Holocene Pelado, Guespalapa and Chichinautzin scoria cones, south of Mexico City: Journal of Volcanology and Geothermal Research, 130, 197-226.

Vázquez-Sánchez, E., Jaimes-Palomera, R., 1989, Geología de la cuenca de México: Geofísica Internacional, 28 (2), 133-190.

Zamorano-Orozco, J.J., Tanarro-García, L.M., Lugo-Hubp, J., SánchezRubio, G., 2002, Evolución geológica y geomorfología del complejo dómico Los Pitos, norte de la Cuenca de México: Revista Mexicana de Ciencias Geológicas, 19 (1), 66-79.

Manuscrito recibido: Octubre 7, 2014.

Manuscrito corregido recibido: Abril 30, 2015.

Manuscrito aceptado: Agosto 6, 2015. 